\title{
Quick survey for detection, identification and characterization of Acanthamoeba genotypes from some selected soil and water samples in Pakistan
}

\author{
Tania Tanveer ${ }^{1,3}$, Abdul Hameed ${ }^{2}$, Asma Gul'3 , Abdul Matin ${ }^{1}$ \\ ${ }^{1}$ Department of Medical Laboratory Technology, University of Haripur, Haripur, Khyber Pakhtunkhwa, Pakistan \\ ${ }^{2}$ Institute of Biomedical and Genetic Engineering, Islamabad, Pakistan \\ ${ }^{3}$ Department of Environmental Sciences, International Islamic University, Islamabad, Pakistan
}

Tanveer T, Hameed A, Gul A, Matin A. Quick survey for detection, identification and characterization of Acanthamoeba genotypes from some selected soil and water samples in Pakistan. Ann Agric Environ Med. 2015; 22(2): 227-230. doi: 10.5604/12321966.1152070

\begin{abstract}
Acanthamoeba is an opportunistic protozoan pathogen which is widely distributed in nature and plays a pivotal role in the ecosystem. Acanthamoeba species may cause blinding keratitis and fatal granulomatous encephalitis involving the central nervous system. This study investigates the presence of Acanthamoeba in soil and water resources in Pakistan. Acanthamoeba were recovered on non-nutrient agar plate lawn with $E$. coli and identified by morphological characteristics of the cyst. PCR was performed with genus-specific primers, followed by direct sequencing of the PCR product for molecular identification. Overall, the PCR and sequencing results confirmed pathogenic genotypes, including T4 and T15, from both soil and water samples. This is the first report of Acanthamoeba isolation from both soil and water resources in Pakistan which may serve as a potential threat to human health across the country.
\end{abstract}

Key words

Acanthamoeba, genotyping, soil resources, water resources, PCR, Pakistan

\section{INTRODUCTION}

Acanthamoeba is free-living protozoan pathogen widely distributed in nature. They could be found in diverse soil, dust, air and water environment $[1,2,3]$. In addition, they have been isolated from vegetables, some animals (fish, reptiles, amphibians, dogs, monkeys and birds), pulmonary secretions, maxillary sinus and stool samples [4], and are known to be one of the most ubiquitous organisms. The Genus Acanthamoeba consists of both pathogenic and non-pathogenic isolates. Given the correct access and host conditions, pathogenic Acanthamoeba can cause serious human diseases, such as eye keratitis, or the rare and lifethreatening Acanthamoeba granulomatous encephalitis $[1,2]$. Given the free-living nature of the organisms, it is anticipated that humans encounter Acanthamoeba during their normal life. The genus Acanthamoeba has been classified into 17 different genotypes (T1-T17) and has been isolated from environmental resources worldwide [5]. To the best of our knowledge, this is the first study demonstrating the isolation and molecular characterization of Acanthamoeba genotypes in soil as well as water resources in Pakistan.

\section{MATERIALS AND METHOD}

Cultures of Acanthamoeba and Escherichia coli. A clinical isolate of Acanthamoeba castellanii belonging to the T4

\footnotetext{
Address for correspondence: Dr. Abdul Matin, PhD, Department of Medical Laboratory Technology, University of Haripur, Hattar Road, Haripur, Khyber Pakhtunkhwa, 22620-Pakistan

E-mail: amawan@live.co.uk

Received: 14 November 2013; accepted: 22 April 2014
}

genotype isolated from a Keratitis patient (ATCC 50492) was used as a control. Acanthamoeba were grown without shaking in $15-\mathrm{ml}$ of PYG medium [proteose peptone $0.75 \%$ $(\mathrm{w} / \mathrm{v})$, yeast extract $0.75 \%(\mathrm{w} / \mathrm{v})$ and glucose $1.5 \%(\mathrm{w} / \mathrm{v})]$ in T-75 tissue culture flasks at $30^{\circ} \mathrm{C}$, as previously described [6]. Gram-negative bacteria E. coli K12 (HB101), which is a non-invasive laboratory strain, were also used in this study. Bacteria were grown overnight in Luria-Bertini (LB) medium, containing $1 \%(\mathrm{w} / \mathrm{v})$ tryptone, $0.5 \%(\mathrm{w} / \mathrm{v})$ yeast extract and $1 \%(\mathrm{w} / \mathrm{v}) \mathrm{NaCl}$, as described previously [7].

Sample collection, processing and Acanthamoeba isolation on nutrient agar plates. A survey was conducted of Acanthamoeba isolation in 20 representative cities of Pakistan. The soil and water samples were collected from different types of resources from January 2012 - December 2012. The total water sample volume of $500 \mathrm{ml}$ was thoroughly mixed and filtrated through a cellulose nitrate filter, as described previously [8]. Each filter was placed upside down on 1.5\% non-nutrient agar plates seeded with heat-killed E. coli (a non-invasive strain HB101). The plates were then incubated at $30^{\circ} \mathrm{C}$ for up to 2 weeks until growth of amoeboid plaques was visible. During this incubation period, amoebic growth was examined daily by inverted phase microscopy. In contrast, soil samples (2 g) were collected and dissolved in $20 \mathrm{ml}$ of distilled sterile water, and $150 \mu \mathrm{l}$ of each sample was inoculated onto $1.5 \%$ non-nutrient agar plates, as described previously [9].

DNA extraction from purified samples obtained from platting assays. Amoeboid plaques were scraped off the agar plates and DNA extraction performed, as described previously $[8,10]$. Briefly, the cells were palleted at $10,000 \mathrm{~g}$ for $5 \mathrm{~min}$ at RT, followed by resuspension in lysis buffer 
[(100mM KCl, 40mM Tris, 5mM MgCl2, 1\% (w/v) Tween-20 and $100 \mu \mathrm{g} / \mathrm{ml}$ proteinase $\mathrm{K})]$. Next, the tubes were incubated for $1 \mathrm{~h}$ at $56^{\circ} \mathrm{C}$, followed by $10 \mathrm{~min}$ incubation at $100^{\circ} \mathrm{C}$ to inactivate proteinase K. Finally, the tubes were centrifuged at $10,000 \mathrm{~g}$ for $5 \mathrm{~min}$ and supernatants collected and used as DNA template. The purity of DNA was determined by using a Hitachi U-3210 spectrophotomer.

Identification of Acanthamoeba using PCR amplifications and genotyping. The DNA amplification reactions were performed using the genus-specific primers, as described previously $[8,10]$. Primer pair includes the forward primer JDP1 (5-GGCCCAGATCGTTTACCGTGAA) and the reverse primer JDP2 (5-TCTCACAAGCTGCTAGGGAGTCA). PCR reaction was performed in $20 \mu \mathrm{l}$ volume containing 1.25

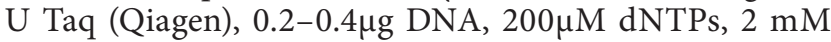
$\mathrm{MgCl}_{2}$ and $2 \mu \mathrm{M}$ primer at $95^{\circ} \mathrm{C}$ for $5 \mathrm{~min}$ for $1 \mathrm{cycle}, 94^{\circ} \mathrm{C}$ for $1 \mathrm{~min}, 60^{\circ} \mathrm{C}$ for $1 \mathrm{~min}$ and $72^{\circ} \mathrm{C}$ for $2 \mathrm{~min}$ for $35 \mathrm{cycles}$, and a final elongation step of $10 \mathrm{~min}$ at $72^{\circ} \mathrm{C}$. Amplified DNA was electrophoresed on $2 \%$ agarose gel, stained with ethidium bromide and visualized under ultraviolet (UV) light. After purification, direct sequencing of PCR product was performed with an automated fluorescence sequencing system (3130 Genetic Analyser, model 627-0040; Applied Biosystems, Tokyo, Japan) with the conserved primer 892C(5'-GTCAGAGGTGAAATTCTTGG) to determine the primary DNA sequence of DF3 of Rns. The DF3 sequence nomenclature was used in this study, as described previously [11].

Accession numbers. Sequences are deposited in the GenBank, Acession Nos. KC778186 - KC778190 and KF035055 - KF035057.

\section{RESULTS}

Overall, 16 of the 17 soil (94.11\%) and 12 of the 13 water samples (92.31\%) were positive for the outgrowth of amoeba on non-nutrient agar plates. In total, 28 of 30 (93.33\%) among them, 10 of 17 (58.82\%) soil and 11 of 13 (84.61\%) water samples, were successfully amplified using Acanthamoeba genus specific primers (in total: 21 of $30(70 \%)$. Alternately, 4 of the $17(23.53 \%)$ soil and 4 of the $13(30.77 \%)$ water cultures containing Acanthamoeba were genotyped based on the DF3 sequence. Results revealed that the isolated Acanthamoeba strains belonged to T4 (84.6\%) and T15 (7.6\%) genotypes (Tab. 1).

Several studies have shown Acanthamoeba isolation from soil and water resources worldwide related to human habitats $(5,12)$; but despite the free-living nature of Acanthamoeba and its presence in various environments, to-date, there is not a single reported case of keratitis or encephalitis infections from Pakistan, which may be attributed due to the lack of expertise and awareness even among the clinician community. Although the authors of the presented study have previously produced an extensive water evaluation survey for the isolation of Acanthamoeba from Khyber Pakhtunkhwa (KPK), Pakistan [8], in the presented study they show for the first time pathogenic (T4 and T15) Acanthamoeba genotypes isolated from soil resources of Pakistan (Fig. 1).

It is worth noting that during this investigation, the T4 genotype was isolated from different water resources
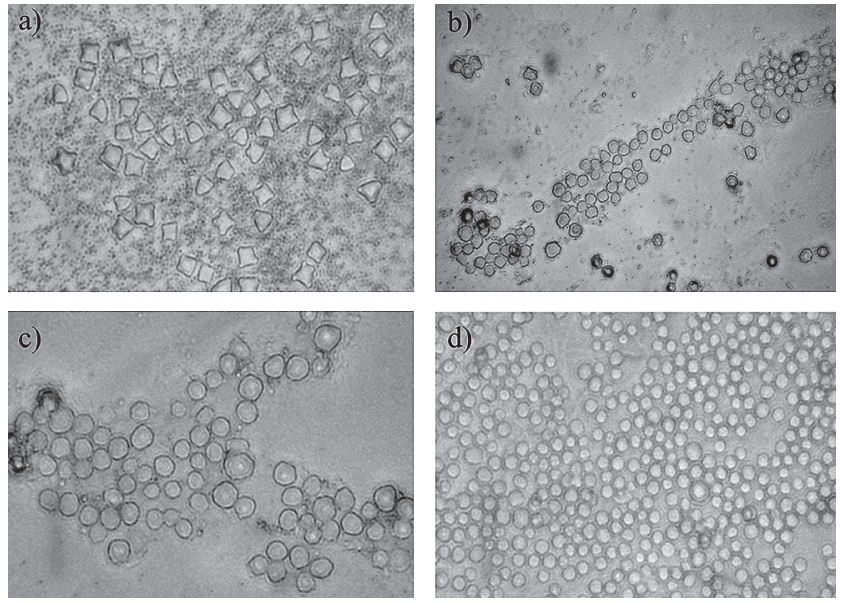

Figure 1. Acanthamoeba cysts $(\times 400)$ on non-nutrient agar plates when observed under inverted microscope. Soil and water samples were pored and filtered, respectively, and inoculated on non-nutrient agar plate lawn with $E$. coli, as described in Materials and methods. Plates were observed for outgrowth up to 14 days and images made. Representative samples (a) KHI-TW4 (b) IBD-TW1 (c) LHR-FS3 (d) KHI-SS2 are shown

in 4 out of $10(40 \%)$ samples, which suggests a very low prevalence of Acanthamoeba T4 genotype in water resources in Pakistan. However, the presence of only a limited number of Acanthamoeba genotypes in the presented study from selected cities across the country is not surprising. The authors have also shown recently the tribal population of KPK has a high titer level of anti-Acanthamoeba antibodies [13], compared with the rest of the country, which supports the finding that Acanthamoeba genotypes are very limited in other parts of the country, except KPK. Overall, the majority of soil and water samples were amplified with Acanthamoeba genus-specific primers, but conclusive sequences could not be obtained and still need to be further investigated. It is speculated that the amplified samples might be the a novel species (Fig. 2), which will be addressed in future studies by the authors. Therefore, the information provided in this study may serve as a base-line for future studies on the role of free-living amoebae, e.g. in outbreaks of water- and soil borne-diseases in the country.

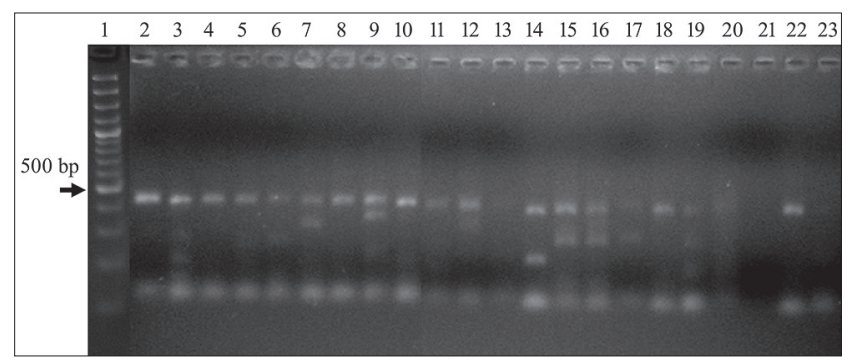

Figure 2. PCR analysis of the amoeba isolated from soil and water samples from representative cities of Pakistan. To confirm the presence of Acanthamoeba populations, DNA were isolated from amoeba recovered from non-nutrient agar plates after 7-14 days and used for PCR analysis using Acanthamoeba-genus specific primers pairs JDP1 and JDP2, as described in Materials and Methods. PCR products were found to be about $500 \mathrm{bp}$ in all samples, confirming the presence of Acanthamoeba. Lane 1: 100 bp DNA ladder; Lane 2: +ve control; Lane 3: IBDTW1; Lane 4: IBD-FS2; Lane 5: KHI-TW4; Lane 6: CHAR-FS4; Lane 7: KHI-SS2; Lane 8: LHR-FS1; Lane 9: LHR-FS3; Lane 10: KHP-TW1; Lane 11: LKA-TW7; Lane 12: MLT-RBS1; Lane 13: LKA-SS7; Lane 14: PSH-BW3; Lane 15: PSH-CW6; Lane 16: PSH-FS9; Lane 17: MUZ-FS2; Lane 18: SRA-TW6; Lane 19: SKD-FS2; Lane 20: SIBI-TW6; Lane 21: WAGA-DS2; Lane 22: QTA-TW3; Lane 23: -ve control 
Table 1. Acanthamoeba genotypes identified in different soil and water resources from representative cities of Pakistan

\begin{tabular}{|c|c|c|c|c|c|c|c|c|c|}
\hline & Sample code & Sources & Sampling area & DNA tube & NNA Culture & PCR & Species & Genotypes & GenBank Accession No \\
\hline 1 & BANU-FS1 & Fertile soil & Bannu & 81 & + & - & ND & - & - \\
\hline 2 & CHAR-FS4 & Fertile soil & Charsadda & 84 & + & + & ND & - & - \\
\hline 3 & DADDO-SS6 & Sandy soil & Daadoo & 67 & + & - & ND & - & - \\
\hline 4 & DIK-DS1 & Deserted soil & Dera Ismail Khan & 74 & + & + & ND & - & - \\
\hline 5 & IBD-TW1 & Tap water & Islamabad & 94 & + & + & Acanthamoeba jacobsi & $\mathrm{T} 15$ & KC778190 \\
\hline 6 & IBD-FS2 & Fertile soil & Islamabad & 95 & + & + & Acanthamoeba sp & - & KC778188 \\
\hline 7 & KHI-TW4 & Tap water & Karachi & 114 & + & + & Acanthamoeba sp & $\mathrm{T} 4$ & KC778187 \\
\hline 8 & KHI-SS2 & Sandy soil & Port Qasim & 103 & + & + & Acanthamoeba jacobsi & T15 & KC778189 \\
\hline 9 & KA-DS1 & Deserted soil & Khyber Agency & 86 & + & - & ND & - & - \\
\hline 10 & KHP-TW1 & Tap water & Khaplu & 111 & + & + & ND & - & - \\
\hline 11 & LHR-FS1 & Fertile soil & Lahore & 14 & + & + & Acanthamoeba sp & T4 & KC778186 \\
\hline 12 & LHR-FS3 & Fertile soil & Lahore & 25 & + & + & Acanthamoeba sp. 1 AM-2013 & $\mathrm{T} 4$ & KF035056 \\
\hline 13 & LHR-TW4 & Tap water & Lahore & 3 & + & + & ND & - & - \\
\hline 14 & LKA-SS7 & Sandy soil & Larkana & 75 & - & - & - & - & - \\
\hline 15 & LKA-TW7 & Tap water & Larkana & 63 & + & + & ND & - & - \\
\hline 16 & MNI-PS2 & Potted Soil & Mianwali & 60 & + & + & ND & - & - \\
\hline 17 & MNI-CW3 & Canal water & Mianwali & 42 & + & + & ND & - & - \\
\hline 18 & MLT-CW3 & Canal water & Multan & 78 & + & + & ND & - & - \\
\hline 19 & MLT-RBS1 & River bank soil & Multan & 99 & + & + & ND & - & - \\
\hline 20 & MUZ-FS2 & Fertile soil & Muzafrabad & 105 & + & - & ND & - & - \\
\hline 21 & PSH-BW3 & Boring water & Peshawar & 104 & + & + & Acanthamoeba jacobsi & $\mathrm{T} 15$ & KF035057 \\
\hline 22 & PSH-CW6 & Canal water & Peshawar & 15 & + & + & Acanthamoeba sp. 2 AM-2013 & - & KF035055 \\
\hline 23 & PSH-FS9 & Fertile soil & Peshawar & 90 & + & + & ND & - & - \\
\hline 24 & SKD-RW1 & River water & Sakardu & 150 & + & - & ND & - & - \\
\hline 25 & SKD-FS2 & Fertile soil & Sakardu & 132 & + & - & ND & - & - \\
\hline 26 & SRA-TW6 & Tap water & Sargodha & 36 & + & + & ND & - & - \\
\hline 27 & SHI-FS2 & Fertile soil & Shinkiari & 18 & + & + & ND & - & - \\
\hline 28 & SIBI-TW6 & Tap water & Sibi & 214 & - & - & - & - & - \\
\hline 29 & QTA-TW3 & Tap water & Quetta & 221 & + & + & ND & - & - \\
\hline 30 & WAGA-DS2 & Deserted soil & Waga & 28 & + & - & ND & - & - \\
\hline
\end{tabular}

ND - Not Determined

\section{CONCLUSIONS}

To the best of the authors' knowledge, this is the first report which demonstrates the presence of Acanthamoeba in the soil and water sources of Pakistan. Pathogenic Acanthamoeba genotypes were identified in fertile (decomposed) soil, which is usually preferred for the cultivation of various crops in Pakistan; this is quite frightening from the public health perspective. In addition, pathogenic Acanthamoeba species were identified from tap water normally consumed by the human population across the country. This, besides confirming the presence of pathogenic species in both soil and water medium, also indicates that they may also serve as a transmission vehicle of pathogenic bacteria in different environmental sources in Pakistan - an additional potential threat to humans. This report is a continuation of the other series of studies which are still underway for the analysis of different environmental sources, such as soil, air and water samples across the country. Further studies are necessary in order to determine the pathogenic potential of the identified genotypes and their role in the environment.
Authors' contributions. AM and TT surveyed the area, collected and processed the samples for amoeba isolation on NNA plates. AH, AG and TT prepared each sample for further molecular analyses, carried out PCR, genotyping, and participated in the interpretation and analysis of data; AM conceived the study, designed the study protocol and carried out interpretation of data and drafted the manuscript. All authors read and approved the final manuscript. AM is guarantor of the paper.

\section{Acknowledgements}

The authors express their thanks to Brig. Muhammad Farooq Murawat, Asim Wazir Khan Naizi, Sohail Khan Naizi, Junaid Khan, Abu Turab Khan and Ghazala Shabnum for their tremendous support in the collection and transportation of samples. This work was partially supported by grants from University of Haripur, Khyber Pakhtunkhwa, Pakistan.

Conflicts of interest. The authors have no conflict of interest to declare. 


\section{REFERENCES}

1. Stehr-Green JK, Baily TM, Visvesvara GS. The epidemiology of Acanthamoeba keratitis in the United States. Am J Ophthalmol. 1989; 107: 331-336.

2. Badenoch PR, Adams M, Coster DJ. Corneal virulence, cytopathic effect on human keratocytes and genetic characterization of Acanthamoeba. Int J Parasitol. 1994; 25: 229-239.

3. Sriram R, Shoff M, Booton G, Fuerst P, Visvesvara GS. Survival of Acanthamoeba cysts after desiccation for more than 20 years. J Clin Microbiol. 2008; 46: 4045-4048.

4. Visvesvara GS, Moura H, Schuster FL. Pathogenic and opportunistic free living amoebae: Acanthamoeba spp., Balamuthia mandrillaris, Negleria fowleri and Sappinia diploidea. FEMS Immun and Med Microbiol. 2007; 50: 1-26.

5. Nuprasert W, Putaporntip C, Pariyakanok L, Jongwutiwes S. Identification of a Novel T17 Genotype of Acanthamoeba from Environmental Isolates and T10 Genotype Causing Keratitis in Thailand. J Clin Microbiol. 2010; 48: 4636-4640.

6. Shoaib HM, Muazzam AG, Mir A, Jung S-Y, Matin A. Evaluation of inhibitory potential of some selective methanolic plants extracts on biological characteristics of Acanthamoeba castellanii using human corneal epithelial cells in vitro. Parasitol Res. 2013; 112 (3): 1179-1188.
7. Matin A, Jung S-Y. Interaction of Escherichia coli K1 and E. coli K5 with Acanthamoeba castellanii trophozoites and cysts. Korean J of Parasitol. 2011; 49: 349-356.

8. Tanveer T, Hameed A, Muazzam AG, Jung S-Y, Gul A, Matin A. Isolation and molecular characterization of potentially pathogenic Acanthamoeba genotypes from diverse water resources including household drinking water from Khyber Pakhtunkhwa, Pakistan. Parasitol Res. 2013; 112 (8): 2925-2932.

9. Lorenzo-Morales J, Monteverde-Miranda CA, Jiménez C, Tejedor ML, Valladares B, Ortega-Rivas A. Evaluation of Acanthamoeba isolates from environmental sources in Tenerife, Canary Islands, Spain. Ann Agric Environ Med. 2005; 12: 233-236.

10. Matin A, Jeong SR, Faull J, Khan NA. Evaluation of prokaryotic and eukaryotic cells as food source for Balamuthia mandrillaris. Arch of Microbiol. 2006; 186: 261-271.

11. Booton GC, Kelly DJ, Chu YW, Seal DV, Houang E, Lam DSC, Byers TJ, Fuerst PA. 18S ribosomal DNA typing and tracking of Acanthamoeba species isolates from corneal scrape specimens, contact lens, lens cases, and home water supplies of Acanthamoeba keratitis patients in Hong Kong. J Clin Microbiol. 2002; 40: 1621-1625.

12. Marciano-Cabral F, Cabral G. Acanthamoeba spp. as agents of disease in humans. Clin Microbiol Rev. 2003; 16: 273-307.

13. Matin A, Ismail M, Mehmood K. Acanthamoeba castellanii. antibody prevalence among diverse tribal Pakistani population. Retrovirology 2012; 9: 47. 